\title{
Efficacy of Trimebutine Maleate in the Treatment of Functional Dyspepsia in Childhood
}

\author{
Nijevitch AA ${ }^{1}$, Akhmadeeva EN², Sataev UV ${ }^{3}$, \\ Idrisov $B^{4}$
}

${ }^{1}$ Dr. Alexander A. Nijevitch, MD, PhD, ${ }^{2}$ Dr. Elsa N. Akhmadeeva MD, PhD, ${ }^{3}$ Dr. Valery U. Sataev MD, PhD, ${ }^{4} \mathrm{Dr}$. Bulat Idrisov MD. All from Bashkortostan State Medical University, Russia.

\section{Introduction}

$\mathrm{P}$ aediatric functional gastrointestinal disorders in childhood include a combination of chronic or recurrent symptoms and are not explained by structural or biochemical abnormalities ${ }^{1}$. The most common complaint among children until the age of 15 is a recurrent abdominal pain (RAP) $)^{2}$. Only in $5-10 \%$ of the children RAP symptom has an underlying organic nature associated with the complaint ${ }^{3}$. Among the children with functional dyspepsia (FD), RAP was determined in $70 \%$ patients $^{2}$. The Rome III committee defined FD as the presence of complex of symptoms including epigastric pain and meal-induced dyspeptic symptoms, comprising a large number of non-painful symptoms (postprandial fullness, early satiety, abdominal bloating, belching and postprandial nausea) in the absence of any organic, systemic or metabolic disease that may explain the symptoms. Any combination of these symptoms may intermittently occur over time ${ }^{4}$. Thus, dyspepsia is an extremely common condition in paediatric practice ${ }^{4}$. Over $50 \%$ of dyspeptic patients in childhood alongside with RAP suffered from nausea, vomiting, bloating, early satiety and nocturnal awakening ${ }^{2,5}$.

Nevertheless, the "dyspepsia" term is non-specific and is often used to describe non-identical symptoms and complaints in different groups of patients. Nowadays, FD remains a clinically important problem in paediatrics associated with considerable health and the experience of significant decrease in quality of life.

The etiology and pathogenesis of FD remain still unclear and have not been fully elucidated ${ }^{21}$.

\section{Address for correspondence \\ Alexander A. Nijevitch \\ PO Box 4894, Ufa-57, 450057, \\ Russia \\ E-mail: aanj@rambler.ru}

\begin{abstract}
Peripheral $\mu$-, k- and $\delta$-opioid agonist trimebutine maleate is considered to be an effective therapeutic drug for the treatment of functional gastrointestinal disorders. Ninety-two paediatric outpatients (1217 year-old) suffering from functional dyspepsia (epigastric pain and meal-induced dyspeptic symptoms) were enrolled in a prospective openlabel study. For ethical reasons, no placebo group was included. Patients were treated with trimebutine maleate (200 $\mathrm{mg}$ three times daily). After a 3-week treatment there was a significant decrease in scores of epigastric pain $(p<0.05)$, postprandial fullness $(p<0.05)$, early satiety $(p<0.05)$, nausea $(p<0.05)$ and belching $(p<0.05)$. The treatment regimen was well tolerated and demonstrated a good compliance. In conclusion, we postulate that trimebutine maleate is an effective medication for relief of main symptoms associated with functional dyspepsia syndrome in childhood. Because of the limited data on therapeutic interventions in functional dyspepsia in childhood and increasing demand for therapies to treat this disorder, further evaluation of the efficacy of trimebutine treatment for children is certain.
\end{abstract}

Key words: Functional dyspepsia, Trimebutine, Opioid agonist, Dyspepsia

Several factors have been proposed in individual patients: delay in gastric and small bowel transit time, decreased postprandial gastric volume accommodation, augmented visceral sensitivity and perception. Disturbed gastrointestinal functions are believed to play an important role in the development of FD-associated symptoms in adolescents ${ }^{5,6}$.

Unfortunately, pharmacological treatments for patients with FD remain unsatisfactory. Standard empirical treatment with antisecretory drugs or prokinetic agents demonstrated heterogeneous results in different clinical trials $s^{7,8,9}$.

\section{How to cite this article?}

Nijevitch AA, Akhmadeeva EN, Sataev UV, Idrisov B. Efficacy of Trimebutine Maleate in the Treatment of Functional Dyspepsia in Childhood. J Nepal Paediatr Soc 2013;33(2):158-162. 
Very limited and conflicting clinical information is available regarding opioid agonists for FD treatment $t^{8,10,11,12}$. Trimebutine maleate (TM), an encephalinergic receptor ligand [3,4,5-trimethoxybenzoic acid 2-(dimethilamino)-2 phenylbutylester] acts as an agonist on peripheral $\mu$, k- and $\delta$ - opiate receptors by triggering phase III of the migrating motor complex ${ }^{13}$. TM has been reported to have a dual action on gastric motility: 1) a stimulatory effect on the hypomotile gastrointestinal tract; 2) an inhibitory effect on the hypermotile tract ${ }^{14}$. TM modifies gastric motility by an increase in frequency of slow wave of peristalsis and blocks cholinergic transmission and $\mathrm{Ca}^{2+}$ influx $1^{5,16}$. Few clinical and experimental studies suggest a beneficial effect of TM on gastric emptying and propulsive electromechanical activity in the gastrointestinal tract ${ }^{14,17}$ and local anesthetic property, which is 17 times more potent than classic drug lidocaine ${ }^{18,19}$. The experience of TM usage in childhood is very restricted, furthermore it is one of the few paediatric drug approved by the Ministry of Health in Russia and the only one approved by Ethical Committee of our hospital where given research was conducted ${ }^{9}$. The aim of this study was to assess the effectiveness of TM for the treatment of paediatric FD patients.

\section{Materials and methods}

This was a prospective, open-label study conducted on February, 2011 through February, 2012. The study was carried out in a tertiary center setting. During the above mentioned period, 161 paediatric outpatients $12-17$ years of age (mean age $13.7 \pm 1.3$ years; 93 girls, 68 boys), who consecutively referred to our unit (Outpatient Department of Children Republican Hospital, Ufa, Russia) with persistent upper abdominal dyspeptic complaints, were considered for the study. The initial evaluation included the standard tests (complete blood count, sedimentation rate, urinalysis, liver and pancreatic profiles, stool cultures and stool examination for ova and parasites). Before endoscopy, pancreatitis, cholecystitis and anatomical abnormalities were excluded using abdominal ultrasonography. All patients or their parents (if subject was younger than 14 years old) approved an informed consent prior to the procedure. The presence of chronic inflammatory bowel disease, lactose malabsorption and celiac disease was assessed by means of standard diagnostic procedures. Esophageal $\mathrm{pH}$ monitoring was performed in selected patients.

Upper gastrointestinal endoscopy is a routine examination for RAP patients with persistent symptoms in our hospital. We used endoscopy to rule out $\mathrm{H}$. pylori associated diseases (gastritis, duodenitis, peptic ulcer disease), esophagitis and celiac disease in the study group.
Endoscopy was performed via an Olympus GIF XP20 endoscope (Olympus Optical, Tokyo, Japan) after overnight fasting and without any prior local medication. During endoscopy, four biopsy samples (from antrum and body) were obtained to estimate the mucosal inflammation and $\mathrm{H}$. pylori presence. Histologic examination of sections was performed with $\mathrm{HE}$ and Giemsa staining. The patients with organic disease (esophagitis, H. pylori-associated gastritis, peptic ulcer disease, pancreatitis, inflammatory bowel disease), or other forms of gastrointestinal functional disorders (irritable bowel syndrome), parasitic infestation (ascariasis, giardiasis) were excluded from the study. Also, the patients were excluded, who had received previous treatment with PPI, bismuth salts, prokinetics, antibiotics, non-steroidal anti-inflammatory drugs (NSAID) or medications known to affect gastrointestinal motility.

Subject inclusion criteria's were three of the following symptoms (recurrent epigastric pain or mealinduced dyspeptic symptoms): postprandial fullness, upper abdominal bloating (and belching), early satiety and nausea with having onset at least 6 months prior to the appointment and presented at least twice a week within the preceding 3 months. So all of the FD patients ( $n=92)$ were included. Trimedat $®$ (trimebutine maleate) (Valenta Pharmaceutica, Shchelokovo, Russia) was administered during 3 weeks at the entry of the study, according to the conditions of use established in the products technical form (100 or $200 \mathrm{mg}$ of TM per tablet). The dose was $200 \mathrm{mg}$ three times daily. Fully informed consent of the patients (or their parents) was obtained for every procedure that was performed. Approval of the study protocol by the Ethics Committee of Children's Republican Hospital was not obtained because the study medication was a commercialized product and was prescribed for approved indications of use. Both verbal and printed instructions were given to all the patients and their parents. The instructions comprised dosage, symptoms assessment scale, possible side effects, et cetera. For ethical reasons, no placebo group was included.

A detailed history and physical examination were obtained from each patient before endoscopy. Information about the symptoms was collected via a dedicated questionnaire completed by children and their parents. The severity of symptoms was graded from 0 to $3(0=$ no symptoms, $1=$ easily tolerable $/ \mathrm{mild}, 2=$ affecting normal daily activities/moderate, and $3=$ preventing normal daily activities/severe). The study included 2 visits. The first visit with initial evaluation was at the study entry. The final visit was performed and examination was repeated in 6 months after the management period completion (after the last dose of the drug). At the end 
of the 3 weeks treatment, all the patients were asked for assessment of compliance and side effects. Compliance was graded as excellent of over $80 \%$ of the provided drug had been used, fair if $60-80 \%$ had been used and poor if less than $60 \%$ had been used.

The significance of difference in categorized data was tested by the Wilcoxon test. For the test, a $p$-value under 0.05 was considered to have statistical significance.

\section{Results}

This was a single center study. The diagnosis of FD was confirmed in 92 patients (57.14\%). Compliance was excellent in the majority of the patients $(83.6 \%, 77$ children), good in $13(14.1 \%)$ and fair in $2(2.2 \%)$ of the patients. There were no side effects or adverse reactions, caused by the drug, which lead to disconfirmation or modification of the treatment regimen. Three patients were lost to follow up.

The management results are presented in Table 1. Response to the treatment can be considered as satisfactory. The best results were obtained in resolution of epigastric pain, postprandial fullness and early satiety, nausea and belching $(p<0.05)$. Complete relief of symptoms occurred in $83(90.22 \%)$ outpatients in the present study.

\section{Discussion}

Functional dyspepsia is a long-lasting gastrointestinal disorder with a very good prognosis ${ }^{22}$. However, recent investigations suggest that many children with RAP demonstrated well response to reassurance, but continue to suffer from the symptoms in adulthood ${ }^{20}$
Optimal initial evaluation of FD syndrome patients is debatable $^{7}$. In our opinion, although ulcer-like symptoms are not reliable as a predicting marker of peptic ulcer, endoscopy is indispensable in separation of organic disease (for example H. pylori infection or celiac disease) from functional gastrointestinal disorder, such as FD. $\mathrm{H}$. pylori infection may play an important role in dyspeptic symptoms development in paediatric patients ${ }^{23}$. At the same time, some previous studies indicate that $\mathrm{H}$. pyloriinfected children cannot be differentiated from those who are not on the basis of their presenting symptoms ${ }^{24}$. Obviously, immediate upper gastrointestinal endoscopy may present the most cost-effective approach in this case. The opposite option is an empiric therapy with antisecretory or prokinetic drugs. Unfortunately, antisecretory drugs $\left(\mathrm{H}_{2}\right.$-receptor antagonists or PPI) can only be helpful with the patients with acid-related dyspeptic symptoms ${ }^{25}$. Since nearly $40 \%$ patients with FD demonstrated delay in gastric emptying, prokinetics is often employed in the FD treatment. Gastric emptying does not response to PPI treatment ${ }^{26}$, and PPIs use in the patients with FD-associated motility disorders will make no sense $e^{4,27}$. At the same time, modern studies do not find any association between gastric emptying and epigastric pain, early satiety, bloating or nausea ${ }^{28}$. Unfortunately, wide use of standard prokinetics such as metoclopramide and domperidone in childhood is very limited owing to their side effects, including dystonic reaction and extrapyramidal disorders ${ }^{29}$. Based on lack of clarity, pharmacotherapy for FD varies widely ${ }^{30}$. Symptom-guided empiric treatment strategies have shown mixed results ${ }^{27}$. The chronic abdominal pain of FD is difficult to treat. PPIs have a significantly smaller effect on visceral pain compared to some prokinetic drugs ${ }^{4}$. Furthermore, recently competent recommendations have been presented, showing that there are no convincing data on the use of prokinetic drugs in children with FD ${ }^{29}$.

Table 1: Comparison of clinical symptoms before and after treatment

\begin{tabular}{|c|c|c|c|c|c|c|c|}
\hline \multirow{2}{*}{ Symptoms } & \multicolumn{3}{|c|}{ Before treatment $(n=92)$} & \multicolumn{3}{|c|}{ After treatment $(n=89)$} & \multirow{2}{*}{$\begin{array}{c}\mathbf{p}^{*}, \\
\text { Wilcoxon test }\end{array}$} \\
\hline & Mild & moderate & severe & mild & moderate & severe & \\
\hline Epigastric pain $(n=91)$ & $\begin{array}{c}12 \\
(13.19 \%)\end{array}$ & $\begin{array}{c}49 \\
(53.84 \%) \\
\end{array}$ & $\begin{array}{c}30 \\
(32.97 \%)\end{array}$ & $\begin{array}{c}8 \\
(8.99 \%)\end{array}$ & $\begin{array}{c}3 \\
(3.37 \%) \\
\end{array}$ & 0 & $<0.05$ \\
\hline $\begin{array}{l}\text { Postprandial fullness/ } \\
\text { upper abdominal } \\
\text { bloating }(n=62)\end{array}$ & $\begin{array}{c}33 \\
(53.23 \%)\end{array}$ & $\begin{array}{c}25 \\
(40.32 \%)\end{array}$ & $\begin{array}{c}4 \\
(6.45 \%)\end{array}$ & $\begin{array}{c}4 \\
(6.45 \%)\end{array}$ & $\begin{array}{c}1 \\
(1.61 \%)\end{array}$ & 0 & $<0.05$ \\
\hline Early satiety $(n=48)$ & $\begin{array}{c}5 \\
(10.42 \%) \\
\end{array}$ & $\begin{array}{c}12 \\
(25.0 \%)\end{array}$ & $\begin{array}{c}31 \\
(64.58 \%)\end{array}$ & $\begin{array}{c}9 \\
(18.75 \%) \\
\end{array}$ & $\begin{array}{c}3 \\
(6.25 \%)\end{array}$ & 0 & $<0.05$ \\
\hline Nausea $(n=31)$ & $\begin{array}{c}7 \\
(22.58 \%)\end{array}$ & $\begin{array}{c}20 \\
(64.52 \%)\end{array}$ & $\begin{array}{c}4 \\
(12.9 \%)\end{array}$ & $\begin{array}{c}3 \\
(9.68 \%)\end{array}$ & 0 & 0 & $<0.05$ \\
\hline Belching $(n=63)$ & $\begin{array}{c}32 \\
(50.79 \%)\end{array}$ & $\begin{array}{c}24 \\
(38.1 \%)\end{array}$ & $\begin{array}{c}7 \\
(11.11 \%)\end{array}$ & $\begin{array}{c}3 \\
(4.76 \%)\end{array}$ & $\begin{array}{c}2 \\
(3.17 \%)\end{array}$ & 0 & $<0.05$ \\
\hline
\end{tabular}

*Significant $p$-values (Wilcoxon test) are for mild, moderate and severe symptoms 
TM has been used in many western countries for the treatment of functional gastrointestinal disorders $^{31}$. TM [2-dimethylamino-2-phenylbutyl-3,4,5trimethoxybenzoat hydrogen maleate] is a non-selective agonist of peripheral $\mu$-, k- and $\delta$-opioid receptors ${ }^{18}$. The mechanisms by which TM is beneficial in FD patients remain incompletely understood ${ }^{32}$. It has been postulated that the association of weak opioid property of TM with sodium channel blockade and strong local anesthetic properties explains the effectiveness of TM in abdominal pain treatment ${ }^{18}$. At the same time ${ }^{1}$, through opiate receptors, TM mediate release of gastrointestinal peptides, such as motilin, and modulation of the release of other peptides, including vasoactive intestinal peptide, gastrin and glucagon ${ }^{33}$. TM has a safe toxicological profile and demonstrates excellent tolerability ${ }^{32}$. There is no evidence to reveal that TM acts at the level of the central nervous system and crosses the blood-brain barrier $^{19}$.

Painful dyspepsia is a main indication for TM as well as irritable bowel syndrome and esophagitis ${ }^{34,35,36,37}$. Recently, a Chinese study ${ }^{10}$ has shown high efficacy of TM in FD treatment. A four week course of TM treatment demonstrated a significant decrease in scores of postprandial fullness, early satiation, abdominal pain and total score of symptoms of FD $(p<0.05)$ in comparison with probiotic management.

TM in our study has revealed an excellent effectiveness in FD symptoms relief in paediatric patients. TM was well tolerated and encouraged good compliance. Unfortunately, our study has several important limitations. The first is a relatively low number of study patients. The second is an open-label design and absence of a placebo group or a group of comparison. Another limitation is a very short followup period. Relief of symptoms was assessed over a 6 months period, whereas FD is a chronic condition often persisting for many years ${ }^{4}$. These limitations should be taken in account when considering the results of our survey.

\section{Conclusion}

Despite all the limitations in our work, we regard further investigations of TM in paediatrics is necessary. It is important to develop a safe, well-tolerated and evidence-based therapy for children suffering from dyspeptic syndrome and recurrent abdominal pain, as well as to provide high level of their quality of life. A universal approach to the treatment of FD-associated symptoms in childhood has not been developed because the pathophysiologic mechanisms of FD are heterogeneous and probably different for children and adults.
Acknowledgement: The authors wish to thank Emil Mingazhev for language assistance and Professors Leyla Akhmadeeva and Steven Sussman for expertise attention and suggestion to this work. Authors are indebted to Professor Ping Sun for his helpful suggestions regarding statistical part of the research.

\section{References}

1. Miele E., Simeone D., Marino A., et al. Functional gastrointestinal disorders in children : an Italian prospective survey. Pediatrics 2004;114:73-78.

2. Spiroglou K., Paroutoglou G., Nikolaides N., et al. Dyspepsia in childhood. Clinical manifestations and management. Ann Gastroenterol 2004;17:173-80.

3. Weydert JA, Ball TM, Davis MF. Systematic review of treatments for recurrent abdominal pain. Pediatrics 2003;111:e1-11.

4. Passos M., Duro D., Fregni F. CNS or classic drugs for the treatment of pain in functional dyspepsia? A systematic review and meta-analysis of the literature. Pain Physician 2008;11:597-609.

5. McOmber M.A., Shulman R.J. Pediatric functional gastrointestinal disorders. Nutr Clin Pract 2008;23:268-74

6. Holtmann G., Talley N.J., Liebregts T., et al. A placebo-controlled trial of itopride in functional dyspepsia. N Engl J Med 2006;354:832-40.

7. Lacy B.E., Weiser K. Gastrointestinal motility disorders: an update. Dig Dis 2006;24:228-42.

8. Hiyama T., Yoshihara M., Matsuo K., et al. Metaanalysis of the effect of prokinetic agents in patients with functional dyspepsia. J Gastroenterol Hepatol 2007;22:304-10.

9. Bekem O., Buyukgebiz B., Aydin A., et al. Prokinetic agents in children with poor appetite. Acta Gastroenterol Belg 2005;68:416-18.

10. Zhong Y.Q., Zhu J., Guo J.N., et al. A randomised and case-control clinical study on trimebutine maleate in treating functional dyspepsia coexisting with diarrhea-dominant irritable bowel syndrome. Zhonghua Nei Ke Za Zhi 2007;46:899-902.

11. Aktas A., Caner B., Ozturk F., et al. The effect of trimebutine maleate on gastric emptying in patients with non-ulcer dyspepsia. Ann Nucl Med 1999;13:231-34.

12. Read N.W., Abitbol J.L., Bardhan K.D., et al. Efficacy and safety of the peripheral kappa agonist fedotozine versus placebo in the treatment of functional dyspepsia. Gut 1997;41:664-68.

13. Barthet $M$, Bouvier M, Pecout C. et al. Effects of trimebutine on sphincter of Oddi motility in patients 
with post-cholecystectomy pain. Alimet Pharmacol Ther 1998;12:647-52.

14. Uchiyama M., Iwafuchi M., Yagi M., et al. Effects of trimebutine on intestinal motility after massive small bowel resection. J Smooth Muscle Res 2000;36:117-26.

15. Takenaga H., Magaribuchi T., Tamaki H. Effects of trimebutine maleate (TM-906) on the smooth muscles of isolated guinea pig gallbladder. Jpn $J$ Pharmacol 1984;35:439-43

16. Xue L., Fukuta H., Yamamoto Y., Suzuki H. Dual effect of trimebutine on electrical responses of gastric smooth muscle in the rat. Eur $J$ Pharmacol 1995;294:75-81.

17. Furukawa K., Kimoto Y. Effects of trimebutine maleate (TM-906) on electrical and mechanical activities of smooth muscles of the guinea-pig stomach. Jpn J Pharmacol 1984;35:307-17.

18. Roman F.J., Lanet S., Hamon J., et al. Pharmacological propetties of trimebutine and $\mathrm{N}$-monodesmethyltrimebutine. JPET 1999; 289: 1391-1397.

19. Fioramonti J., Bueno L. Centrally acting agents and visceral sensitivity. Gut 2002;51 (Suppl 1):i91-i95.

20. Ball T.M., Weydert J.A. Methodological challenges to treatment trials for recurrent abdominal pain in children. Arch Pediatr Adolesc Med 2003;157:1121127.

21. Lozano R., Concha M.G.P., Montealegre A., et al. Effectiveness and safety of levosulpiride in the treatment of dismotility-like functional dyspepsia. Ther Clin Risk Management 2007;3:149-55.

22. Heikinen M., Färkkilä M. What is the long-term outcome of the different subgroups of functional dyspepsia? Aliment Pharmacol Ther 2003;18:22329.

23. Nijevitch A.A., Shcherbakov P.L. Helicobacter pylori and gastrointestinal symptoms in school children in Russia. J Gastroenterol Hepatol 2004;19:490-96.

24. Macarthur C., Saunders N., Feldman W. Helicobacter pylori, gastroduodenal disease, and recurrent abdominal pain in children. JAMA 1995;273:729-34.

25. Armstrong D., Kazim F., Gervais M., Pyzyk M. Early relief of upper gastrointestinal dyspeptic symptoms: a survey of empirical therapy with pantoprazole in Canadian clinical practice. Can J Gastroenterol 2002;16:439-50.
26. Kamiya T., Nagao T., Andou T., et al. Effects of trimebutine maleate on gastric motility in patients with gastric ulcer. J Gastroenterol 1998;33:823-27.

27. Hession P.T., Malagelada J.R. Review article: the initial management of uninvestigated dyspepsia in younger patients - the value of symptomguided strategies should be reconsidered. Aliment Pharmacol Ther 2000;14:379-88.

28. Talley N.J., Locke III G.R., Lahr B.D., et al. Functional dyspepsia, delayed gastric emptying, and impaired quality of life. Gut 2006;55:933-39.

29. Whitifield K.L., Shulman R.J. Treatment options for functional gastrointestinal disorders: from empiric to complementary approaches. Pediatr Ann 2009;38:288-94.

30. Hongo M., Kanatsuka H., Sugawara A., et al. Primary care in the treatment of functional gastrointestinal symptoms in Japan: prescription preferences and impression of results. Aliment Pharmacol Ther 2005;21(Suppl. 2):47-54.

31. Gottrand F. Prokinetics in childhood. Arch Pediatr 2010;17:737-38.

32. Kuiken S.D., Tytgat G.N., Boeckxstaens G.E. Review article: drugs interfering with visceral sensitivity for the treatment of functional gastrointestinal disorders - the clinical evidence. Aliment Pharmacol Ther 2005;21:633-51.

33. Delvaux M., Wingate D. Trimebutine: mechanism of action, effects on gastrointestinal function and clinical results. J Int Med Res 1997;25:225-46.

34. Schuurkes J., Helsen L., Van Nueten J. A comparative study on the effects of domperidone, metoclopramide, clebopride and trimebutine on the gastro-duodenal preparation of the guinea pig. Jpn J Pharmacol 1985;39:123-30.

35. Kountouras J., Zavos C., Chatzopoulos D. H. pylori infection and reflux oesophagitis. Gut 2004;53:912.

36. Shi Z.M., Zhu Y.S., Wang Q.X., Lei M.N. Comparative study on irritable bowel syndrome treated with acupuncture and western medicine. Zhonqque Zhen Jiu 2011;31: 607-609.

37. Poynard T., Regimbeau C., Benhamou Y. Metaanalysis of smooth muscle relaxants in the treatment of irritable bowel syndrome. Aliment Pharmacol Ther 2001;15:355-61. 